10,05

\title{
Динамика решетки и электронная структура кобальт-титановой шпинели $\mathrm{Co}_{2} \mathrm{TiO}_{4}$
}

\author{
(C) М.А. Просников ${ }^{1}$, А.Д. Молчанова ${ }^{2}$, Р.М. Дубровин ${ }^{1}$, К.Н. Болдырев ${ }^{2}$, А.Н. Смирнов ${ }^{2}$, \\ В.Ю. Давыдов ${ }^{1}$, А.М. Балбашов ${ }^{3}$, М.Н. Попова ${ }^{2}$, Р.В. Писарев ${ }^{1}$ \\ ${ }^{1}$ Физико-технический институт им. А.Ф. Иофффе РАН, \\ Санкт-Петербург, Россия \\ ${ }^{2}$ Институт спектроскопии РАН, \\ Троицк, Москва, Россия \\ ${ }^{3}$ Московский энергетический институт, \\ Москва, Россия \\ E-mail: yotungh@gmail.com
}

(Поступила в Редакцию 8 июня 2016 г.)

\begin{abstract}
Представлены результаты исследований фононных возбуждений и электронной структуры обращенной шпинели $\mathrm{Co}_{2} \mathrm{TiO}_{4}$, в которой ниже $T_{C}=56 \mathrm{~K}$ магнитоупорядоченные ионы кобальта $\mathrm{Co}^{2+}\left(3 d^{7}\right)$ находятся в равном количестве в тетраэдрической и октаэдрической подрешетках. Исследовались монокристаллы с использованием оптических методов отражения и поглощения в широком спектральном интервале, рамановского рассеяния, а также метода диэлектрической спектроскопии. Изучена динамика инфракрасных и Раманактивных фононов, обнаружены особенности, связанные с наличием разупорядочения в тетраэдрических позициях. Зарегистрированные $d-d$-электронные переходы в области 3800 и $6300 \mathrm{~cm}^{-1}$ подтверждают координационные особенности ионов $\mathrm{Co}^{2+}$. Обнаружен рост диэлектрической проницаемости в области температур ниже $130 \mathrm{~K}$.
\end{abstract}

Работа выполнена при поддержке Российского научного фонда (проект № 16-12-10456).

\section{1. Введение}

Минерал шпинель $(\mathrm{Mg})\left[\mathrm{Al}_{2}\right] \mathrm{O}_{4}$ с кубической структурой известен и применяется в различных сферах уже более века [1], в том числе как драгоценный камень, один из лучших образцов которого находится в английской короне. В 50-е годы XX века были синтезированы искусственные материалы со структурой шпинели, которые составили важную группу магнитных диэлектриков. В этой структуре тетраэдрические $(\mathrm{Mg})$ и октаэдрические [Al] позиции могут быть заполнены как магнитными ионами (в первую очередь $\mathrm{Fe}$ ), так и ионами $3 d$-группы Периодической таблицы, а также диамагнитными ионами $\mathrm{Zn}$ и др. Возможно, наиболее интересным с физической точки зрения материалом является шпинель-магнетит $\mathrm{Fe}_{3} \mathrm{O}_{4}$, в которой ионы $\mathrm{Fe}^{2+}$ и $\mathrm{Fe}^{3+}$ занимают как октаэдрические, так и тетраэдрические позиции. С одной стороны, в магнетите наблюдаются разнообразные физические явления, например переход металл-диэлектрик, а с другой - магнетит важен с практической точки зрения как руда для выплавки железа. Обменное взаимодействие между магнитными ионами в двух подрешетках шпинели является антиферромагнитным, что в силу соотношения $1: 2$ между числом позиций приводит к результирующему ферримагнитному моменту большинства магнитных шпинелей. Эти свойства можно изменять в широких пределах путем введения в обе магнитные позиции различных магнитных и диамагнитных ионов. Ферримагнитные шпинели являются важными представителями класса материалов, объединенных общим термином „ферриты“, куда входят также ферриты-гранаты, гексаферриты и др. Разнообразие свойств ферритов-шпинелей и их практических приложений необычайно велико, что отражено в многочисленных статьях, монографиях и справочной литературе (см., например, [2-5]).

Среди многочисленных оксидных ферритов-шпинелей с разным составом и, следовательно, физическими свойствами можно выделить шпинель (Co) $\left[\mathrm{CoTi}^{\circ} \mathrm{O}_{4}[6]\right.$. Ее особенностью является то, что магнитные ионы $\mathrm{Co}^{2+}$ (оболочка $3 d^{7}$ ) входят в равном количестве в тетраэдрические и октаэдрические позиции, т.е. этот материал является обращенной (inverse) шпинелью. Поскольку ионы титана $\mathrm{Ti}^{4+}$ (оболочка $3 d^{0}$ ) являются диамагнитными, можно было бы предполагать, что магнитные моменты ионов кобальта $\mathrm{Co}^{2+}$ в двух подрешетках должны компенсировать друг друга и магнитный момент должен отсутствовать. Тем не менее ферримагнитный момент наблюдался ниже температуры Кюри $T_{C}=56 \mathrm{~K}$ [6]. Уже это первое исследование показало необычное поведение намагниченности при понижении температуры ниже $T_{C}$. Сначала наблюдался рост намагниченности, но при температуре около $30 \mathrm{~K}$ намагниченность обращалась в нуль, т. е. имела место точка магнитной компенсации, а ниже этой температуры направление оси легкого намагничивания оказывалось противоположным приложенному внешнему магнитному полю. Дальнейшие магнитные и нейтронно-дифракционные исследования показали, что основное магнитное состояние этой обращенной шпинели представляет собой наложение продольной ферри- 
магнитной компоненты и поперечной компоненты типа спинового стекла [7]. Следует, однако, отметить, что в недавних сообщениях были высказаны предположения, что в октаэдрической позиции ионы кобальта и титана имеют валентность $\left[\mathrm{Co}^{3+} \mathrm{Ti}^{3+}\right][8]$, в то время как в аналогичной шпинели $\mathrm{Co}_{2} \mathrm{SnO}_{4}$ ионы в октаэдрической позиции имеют валентность $\left[\mathrm{Co}^{2+} \mathrm{Sn}^{4+}\right]$ [9]. Можно заключить, что вопрос о микроскопической природе этой намагниченности остается открытым.

Необычность магнитного поведения шпинели $\mathrm{Co}_{2} \mathrm{TiO}_{4}$ и имеющаяся неоднозначность в валентном и, следовательно, магнитном состояниях анионов кобальта и титана дают все основания для постановки исследований электронной структуры этого материала с привлечением оптических спектроскопических методов. Хотя данный материал известен уже довольно давно [6], сообщения об изучении его оптических свойств в литературе полностью отсутствуют. Можно предположить, что это связано с тем, что большинство исследований было выполнено на поликристаллических и керамических образцах. Совсем недавно было опубликовано сообщение об оптическом исследовании наночастиц $\mathrm{Co}_{2} \mathrm{TiO}_{4}$, но анализа фононной и электронной структур проведено не было [10]. Можно еще добавить, что спектроскопические исследования в видимой области спектра имеют важность ввиду того, что шпинель $\mathrm{Co}_{2} \mathrm{TiO}_{4}$ является представителем группы экологически чистых (environmentfriendly) коммерческих зеленых пигментов [11].

В настоящей работе сообщается о выращивании монокристаллов $\mathrm{Co}_{2} \mathrm{TiO}_{4}$ и результатах их исследования с использованием спектроскопических методов отражения и поглощения света в широком спектральном диапазоне, а также комбинационного (рамановского) рассеяния света. В дополнение к оптическим исследованиям нами также изучена температурная зависимость диэлектрической проницаемости в температурной области $4.2-350 \mathrm{~K}$ и частотном интервале $10 \mathrm{kHz}-1 \mathrm{MHz}$.

\section{2. Теоретические аспекты}

2.1. Кристаллическая структура и фононные моды. Соединение $\mathrm{Co}_{2} \mathrm{TiO}_{4}$ кристаллизуется в кубической пространственной группе $F d \overline{3} m$ (№ 227, $Z=8)$, точечная группа $m \overline{3} m$ [8]. Параметр решетки $a=8.45 \AA . \mathrm{Co}_{2} \mathrm{TiO}_{4}$ является обращенной шпинелью, в которой ионы $\mathrm{Co}^{2+}$ заполняют все тетраэдрические позиции $8 a$ с позиционной симметрией $\overline{4} 3 m$ и половину октаэдрических позиций $16 d(. \overline{3} m)$, в то время как оставшиеся позиции $16 d$ заполняют ионы $\mathrm{Ti}^{4+}$. Ионы кислорода расположены в позициях $32 e(, 3 m)$. Кристаллическая структура изображена на рис. $1 . \mathrm{Co}_{2} \mathrm{TiO}_{4}$ является ферримагнетиком; при температурах ниже $T_{C}=56 \mathrm{~K}$ происходит ферримагнитное упорядочение с точкой компенсации магнитного момента $T_{\text {comp }}=32 \mathrm{~K}$. При дальнейшем понижении температуры наблюдается магнитный момент, величина которого зависит от маг-

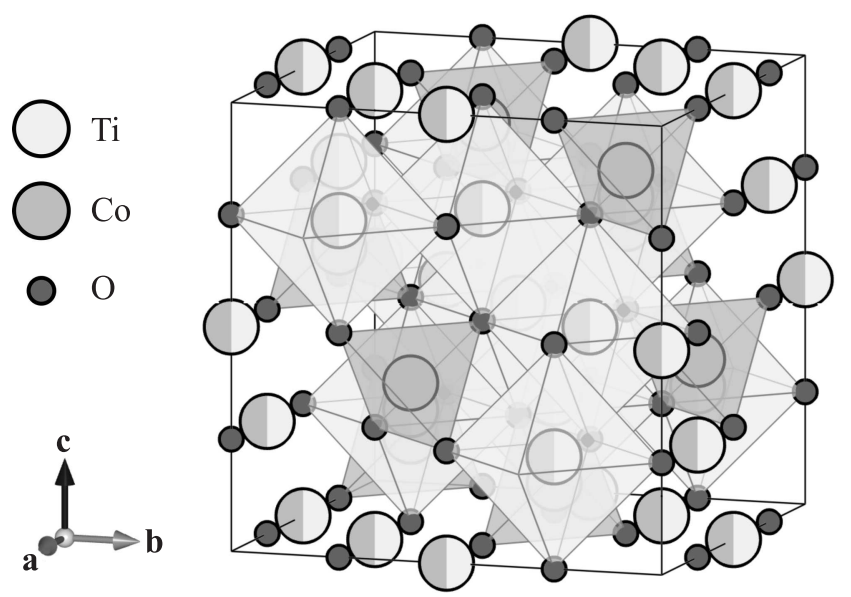

Рис. 1. Кристаллическая структура кобальт-титановой шпинели $\mathrm{Co}_{2} \mathrm{TiO}_{4}$. Октаэдрические позиции заняты ионами [Co/Ti] (обозначены двухцветными кружками).

нитного поля, насыщение не было обнаружено вплоть до полей 15 T [8]. Также сообщалось о наблюдении несоразмерных магнитных структур, состоянии спинового стекла [7] и наличии магнитных кластеров [8,9]. Указанные эффекты, вероятно, вызваны разупорядочением ионов $[\mathrm{Co} / \mathrm{Ti}]$ в октаэдрических позициях.

Теоретико-групповой анализ предсказывает следующий набор фононных мод в центре зоны Бриллюэна (Г-точка):

$$
\Gamma=A_{1 g}+3 A_{2 u}+3 E_{u}+E_{g}+3 T_{2 u}+3 T_{2 g}+7 T_{1 u}+T_{1 g} .
$$

После вычитания акустической моды $T_{1 \text { u }}$ остается 21 мода оптических фононов, среди которых имеется пять $\left(A_{1 g}+E_{g}+3 T_{2 g}\right)$ четных Раман-активных мод. Моды $6 T_{1 u}$ полярны и активны в инфракрасном (ИК) поглощении/отражении, а оставшиеся моды неактивны. Согласно правилам отбора, $\left(A_{1 g}+E_{g}\right)$-фононы активны в параллельных поляризациях вдоль направлений $\langle 100\rangle$, в то время как оставшиеся $T_{2 g}$-моды должны наблюдаться в скрещенных поляризациях. Для ИК-активных фононов $T_{1 u}$ условием наблюдения является $E \|\langle 100\rangle$. Следует ожидать различия в ИК-спектрах обращенной и нормальной (ионы одного типа находятся только в одной из позиций) шпинели, а именно наличия шести против четырех $T_{1 u}$-мод, что связано с заполнением позиций $16 d$ ионами двух типов. Указанные позиции центросимметричны, и, следовательно, расположенные в них ионы не будут вносить вклад в комбинационное рассеяние.

2.2. Электронная структура иона $\mathrm{Co}^{2+}\left(3 d^{7}\right)$ в тетраэдрическом и октаэдрическомкристаллических полях. В типичной ферримагнитной шпинели, например в магнетите $\mathrm{Fe}_{3} \mathrm{O}_{4}$, или иначе $\left(\mathrm{Fe}^{2+} \mathrm{O}\right)\left[\mathrm{Fe}_{2}^{3+} \mathrm{O}_{3}\right]$, двухвалентные и трехвалентные магнитные ионы занимают в элементарной ячейке тетраэдрические и октаэдрические позиции соответственно. 


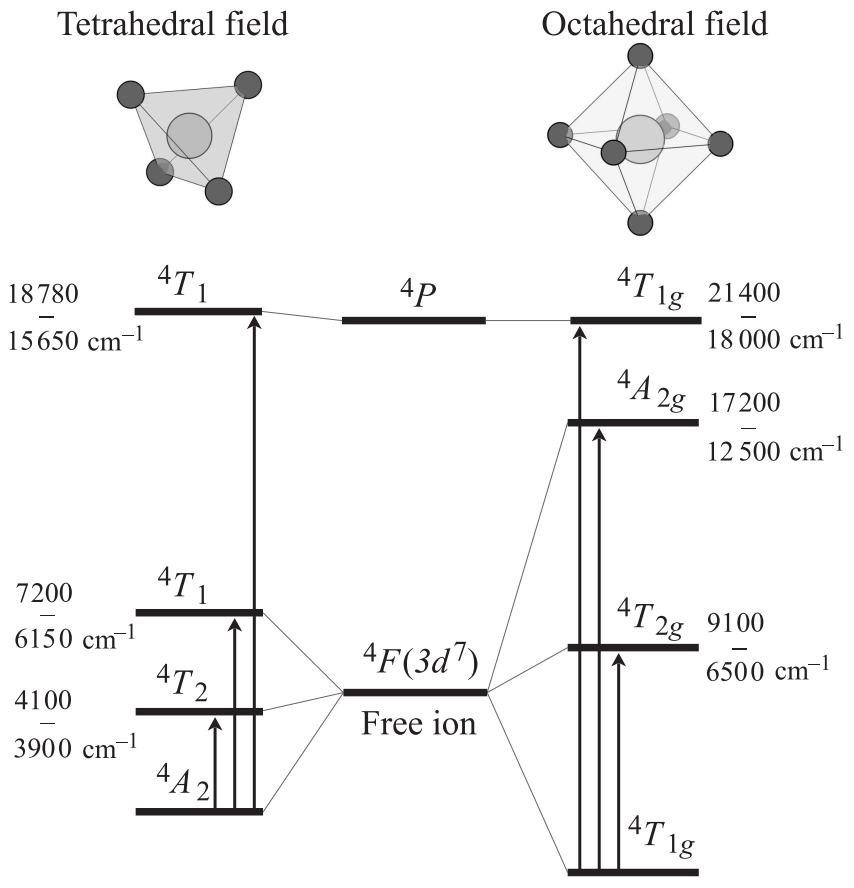

Рис. 2. Электронная схема расщепления уровней иона $\mathrm{Co}^{2+}$ $\left(3 d^{7}\right)$ в тетраэдрическом (слева) и октаэдрическом (справа) кристаллических полях для высокоспинового состояния $S=3 / 2$. Диапазоны энергий электронных переходов указаны для различных соединений, приведенных в табл. 5.18 в монографии [12].

Указанное распределение заряда является, вероятно, причиной таких свойств, как высокая проводимость и низкое удельное сопротивление многих шпинелей, а также возможности переходов металл-диэлектрик и других явлений. Особенностью монокристаллов шпинели $(\mathrm{Co})[\mathrm{CoTi}] \mathrm{O}_{4}$ является высокое удельное сопротивление, превышающее $100 \mathrm{M} \Omega$, и это дает нам основание полагать, что как тетраэдрические, так и половина октаэдрических позиций заполнены ионами одной валентности, а именно $\mathrm{Co}^{2+}$.

На рис. 2 приведена схема расщепления электронных уровней для иона $\mathrm{Co}^{2+}$ в тетраэдрическом (позиция $8 a$ ) и октаэдрическом (позиция 16d) кристаллических полях. В тетраэдрических позициях основным состоянием является орбитальный синглет ${ }^{4} A_{2}$, и в силу отсутствия центра инверсии переходы с него на возбужденные уровни разрешены в электродипольном приближении. Это обстоятельство предполагает высокую интенсивность соответствующих электронных переходов. С другой стороны, в октаэдрических позициях с центром инверсии основным состоянием является орбитальный триплет и, следовательно, в них запрещены электродипольные переходы. Кроме того, орбитальный триплет предполагает существенный вклад орбитального момента в суммарный магнитный момент иона $\mathrm{Co}^{2+}$ в этой позиции. Отсутствие насыщения ниже $T_{C}$ в полях вплоть до $15 \mathrm{~T}$ [8] также подтверждает наличие значительного орбитального вклада в магнитный момент в октаэдрической решетке.

\section{3. Детали эксперимента}

3.1. Выращивание монокристаллов . Монокристаллы соединения $\mathrm{Co}_{2} \mathrm{TiO}_{4}$ выращивались методом бестигельной зонной плавки с радиационным (световым) нагревом на аппаратуре типа УРН-2-ЗП (НИУ МЭИ). В качестве ориентированных затравок были использованы стержни размером $3 \times 3 \times 12 \mathrm{~mm}$, вырезанные из монокристаллов марганец-цинковой феррошпинели или из выращенных кристаллов Со-Ті-шпинели. Поликристаллические заготовки для выращивания кристаллов приготавливались с использованием обычных методов керамической технологии из реактивов-оксидов квалификации осч. Монокристаллы $\mathrm{Co}_{2} \mathrm{TiO}_{4}$ были выращены со скоростью $8 \mathrm{~mm} / \mathrm{h}$ при вращении кристалла и заготовки 40 и $2 \mathrm{rpm}$ соответственно. Процесс роста проводился в атмосфере воздуха.

Исследование поверхности срезов монокристаллов и определение состава кристалла и включений проводились с использованием сканирующего электронного микроскопа JSM5910-LV с приставкой для анализа дифракции отраженных электронов Inca Crystal и энергодисперсионного рентгеновского микроанализатоpa Oxford Instrument Analytical. Этот высокочувствительный метод исследования поверхности не выявил каких-либо фазовых включений и неоднородностей типа пустот и пр. по всей поверхности исследуемого образца диаметром $\sim 10 \mathrm{~mm}$. На рис. 3 показана лауэграмма монокристалла $\mathrm{Co}_{2} \mathrm{TiO}_{4}$, свидетельствующая о том, что объем монокристалла состоит из фазы $\mathrm{Co}_{2} \mathrm{TiO}_{4}$. Узкие дифракционные пики указывают на высокое структурное совершенство кристалла.

3.2. Комбинационное рассеяние. Спектры комбинационного рассеяния в области $90-2000 \mathrm{~cm}^{-1}$ были изучены с использованием спектрометра Т64000 (Jobin-Yvon), оборудованного охлаждаемой жидким азотом CCD-камерой. В качестве источника возбуждения использовался непрерывный $\mathrm{Nd}$ : YAG-лазер с дли-

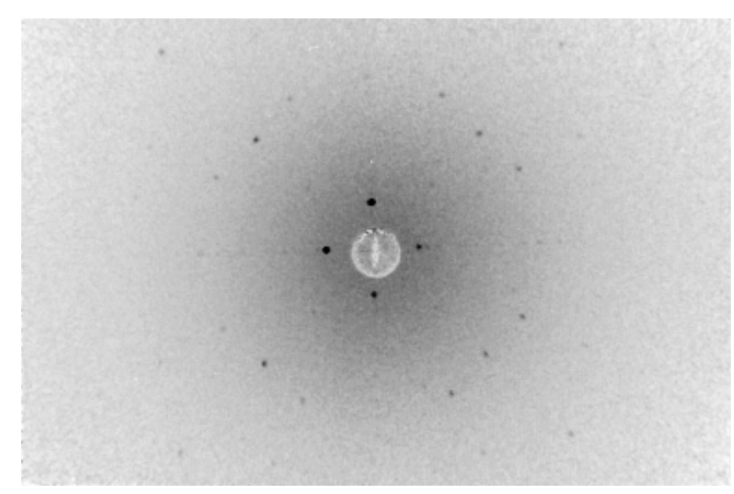

Рис. 3. Лауэграмма монокристалла $\mathrm{Co}_{2} \mathrm{TiO}_{4}$. 
ной волны излучения $532 \mathrm{~nm}(2.33 \mathrm{eV})$. Для фокусировки и сбора рассеянного излучения применялся объектив $50 \times$, размер пятна на образце не превышал $50 \mu \mathrm{m}$. Для исключения перегрева образца мощность лазерного излучения была не больше $3 \mathrm{~mW}$. Охлаждение и термостабилизация образца в интервале температур $10-300 \mathrm{~K}$ осуществлялись в гелиевом криостате замкнутого цикла. Все измерения проведены в геометрии обратного рассеяния при параллельных и скрещенных поляризациях.

3.3. Оптическая спектроскопия отражения и поглощения. Спектры отражения и пропускания монокристаллов $\mathrm{CoTi}_{2} \mathrm{O}_{4}$ в видимом, а также ближнем, среднем и дальнем ИК-диапазонах были зарегистрированы на Фурье-спектрометре высокого разрешения Bruker IFS $125 \mathrm{HR}$ со спектральным разрешением $1-10 \mathrm{~cm}^{-1}$. Измерение спектров пропускания проводилось в диапазоне температур $3.5-300 \mathrm{~K}$ с помощью криостата замкнутого гелиевого цикла Cryomech ST403.

3.4. Диэлектрические измерения. Диэлектрические свойства измерялись с помощью прецизионного измерителя RLC (АКТАКОМ AM 3028) в частотном диапазоне от $10 \mathrm{kHz}$ до $1 \mathrm{MHz}$. Температурные измерения проводились с использованием криостата Oxford Microstat $\mathrm{He} 2$ в диапазоне от 4.2 до $350 \mathrm{~K}$. В качестве электродов использовались слои серебряной пасты.

\section{4. Экспериментальные результаты и их обсуждение}

4.1. Комбинационное рассеяние. В полносимметричных $a(b b) \bar{a}$ спектрах комбинационного рассеяния, показанных на рис. 4, при комнатной температуре наблюдаются два интенсивных фонона с частотами 531.8 и $702.2 \mathrm{~cm}^{-1}$. В спектрах при поляризациях $a(b c) \bar{a}$ (рис. 5) наблюдаются слабые фононы, число которых превышает предсказанное теорией групп, что, возможно, связано с разупорядочением в позициях $16 d$. Наличие ионов $\mathrm{Co}^{2+}$ и $\mathrm{Ti}^{4+}$ различной массы в октаэдрических позициях также приводит к увеличению ширин фононных линий. Можно также отметить „протечки“ интенсивных $T_{2 g^{-}}$и $A_{1 g}$-фононов, что, вероятно, связано с деполяри-

Таблица 1. Частоты и ширины на половине высоты (FWHM) Раман-активных фононов при 300 и $10 \mathrm{~K}$ (для сравнения приведены частоты фононов обращенной шпинели $\mathrm{Fe}_{3} \mathrm{O}_{4}$ [13])

\begin{tabular}{|c|c|c|c|c|c|}
\hline \multirow{2}{*}{ Мода } & \multicolumn{2}{|c|}{ Частота, $\mathrm{cm}^{-1}$} & \multicolumn{2}{|c|}{ FWHM, $\mathrm{cm}^{-1}$} & \multirow{2}{*}{$\begin{array}{c}\text { Частота } \\
\mathrm{Fe}_{3} \mathrm{O}_{4}[13], \mathrm{cm}^{-1}\end{array}$} \\
\hline & $300 \mathrm{~K}$ & $10 \mathrm{~K}$ & $300 \mathrm{~K}$ & $10 \mathrm{~K}$ & \\
\hline$T_{2 g}$ & 182.9 & 187.4 & 9 & 6 & 180 \\
\hline$E_{g}$ & 309.5 & 314.2 & 52 & 47 & 306 \\
\hline$T_{2 g}^{\delta}$ & 475.4 & 481.4 & 39 & 28 & $450-490$ \\
\hline$T_{2 g}$ & 531.8 & 535.2 & 81 & 72 & 538 \\
\hline$A_{1 g}$ & 702.2 & 704.2 & 35 & 29 & 668 \\
\hline
\end{tabular}

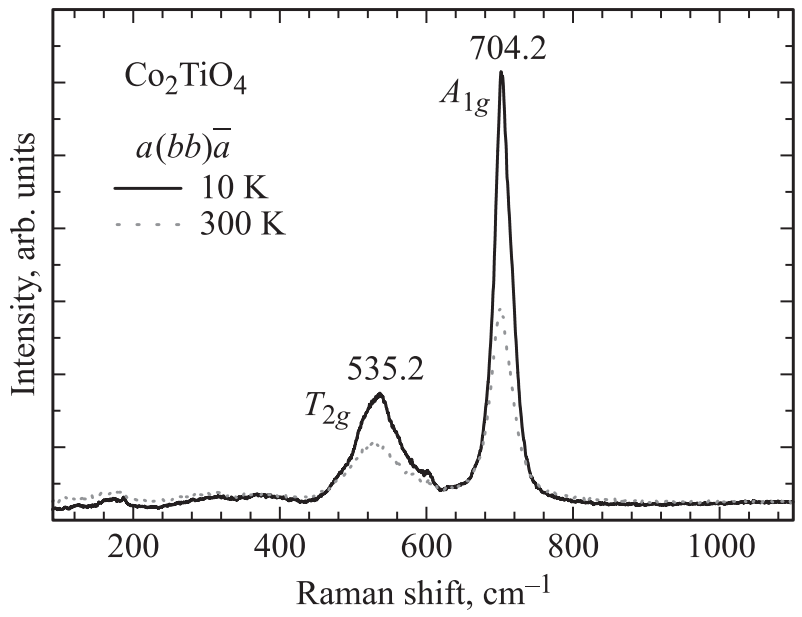

Рис. 4. Спектр комбинационного рассеяния при параллельных поляризациях для температур 10 и $300 \mathrm{~K}$. Числа - частоты фононов $\left(\mathrm{cm}^{-1}\right)$.

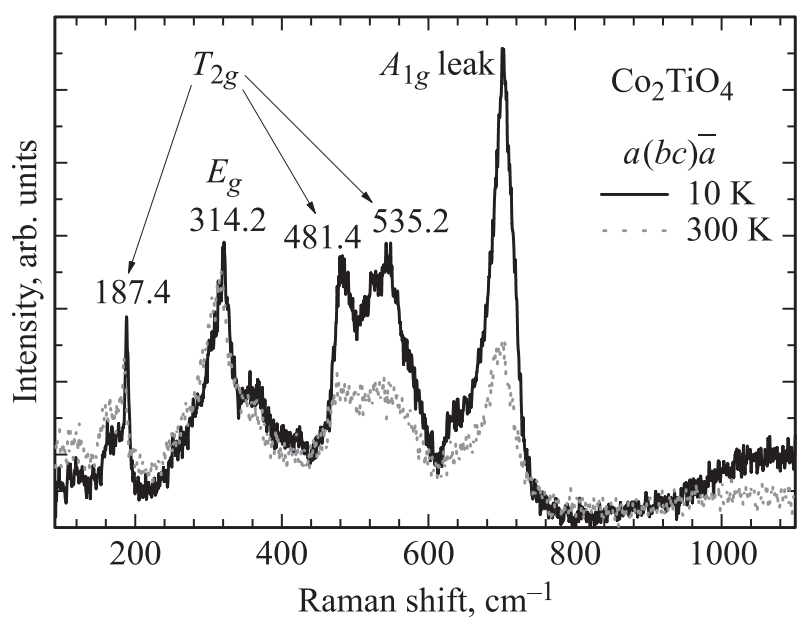

Рис. 5. Спектр комбинационного рассеяния $\mathrm{Co}_{2} \mathrm{TiO}_{4}$ в скрещенных поляризациях для температур 10 и $300 \mathrm{~K}$. Числа частоты фононов $\left(\mathrm{cm}^{-1}\right)$.

зацией на оптических элементах и отклонением поляризации от направления $\langle 100\rangle$. Этот результат затрудняет однозначное отнесение наблюдаемых возбуждений к конкретным фононам. Частоты наиболее интенсивных фононов при 10 и $300 \mathrm{~K}$ приведены в табл. 1 в сравнении с данными для шпинели-магнетита $\mathrm{Fe}_{3} \mathrm{O}_{4}$ [13].

Можно предполагать, что отклонение от правил отбора и присутствие запрещенного фонона $T_{2 g}$ в спектрах поляризации $a(b b) \bar{a}$, а также наличие фонона $E_{g}$ в спектрах $a(b c) \bar{a}$ вызваны особенностями обращенных шпинелей, а именно наличием ионов $[\mathrm{Co} / \mathrm{Ti}]$ в октаэдрических позициях. Следует отметить, что в литературе имеются неоднозначные трактовки наблюдаемых фононных мод как в нормальных, так и в обращенных шпинелях [13-15]. На температурных зависимостях спектров наблюдается сдвиг фононов в область высоких частот на $2-6 \mathrm{~cm}^{-1}$, связанный с уменьшением роли ангармонизма и тепловым сжатием решетки [16]. Следует 
отметить, что заметных фононных аномалий в области установления ферримагнитного упорядочения $T=56 \mathrm{~K}$ и вблизи температуры компенсации $T_{\text {comp }}=30 \mathrm{~K}$ обнаружено не было.

4.2. Оптическая спектроскопия отражения и поглощения. В ИК-области частот $100-800 \mathrm{~cm}^{-1}$ были зарегистрированы шесть фононов симметрии $T_{1 u}$ в согласии с теоретико-групповым анализом в случае заполнения октаэдрических позиций разными ионами (рис. 6). Параметры ИК-активных фононных мод были определены с помощью моделирования спектров отражения с использованием программы RefFIT [17]. Подгонка спектров осушествлялась методом наименьших квадратов по формуле

$$
R(\omega)=\left|\frac{\sqrt{\varepsilon(\omega)}-1}{\sqrt{\varepsilon(\omega)}+1}\right|^{2},
$$

где $R(\omega)$ - коэффициент отражения, $\varepsilon(\omega)-$ функция диэлектрической проницаемости, представленная в виде суммы затухающих осцилляторов

$$
\varepsilon(\omega)=\varepsilon_{\infty}+\sum_{j=1}^{J} \frac{f_{j} \omega_{j}^{2}}{\omega_{j}^{2}-\omega^{2}+i \gamma_{j}^{2} \omega} .
$$

Здесь $J-$ полное число осцилляторов, а $\omega_{j}, f_{j}$ и $\gamma_{j}-$ частота, сила и затухание $j$-го осциллятора соответственно. Указанные параметры приведены в табл. 2. Отметим, что наши результаты по частотам фононов в монокристаллах $\mathrm{Co}_{2} \mathrm{TiO}_{4}$ значительно отличаются от результатов, полученных на нанокристаллах [10].

Спектральная область частот 2000-15000 $\mathrm{cm}^{-1}$ характеризуется очень интенсивным поглощением, обусловленным электронными переходами между $3 d$-подуровнями иона $\mathrm{Co}^{2+}$ в тетраэдрических и октаэдрических позициях. Переходы в тетраэдрических позициях без центра инверсии являются разрешенными в электродипольном приближении, и поэтому можно обоснованно полагать, что они должны иметь более высокую интенсивность по сравнению с запрещенными переходами в октаэдрических позициях (рис. 2). Спектры пропускания были измерены при комнатной температуре на кристаллах с толщинами 16 и $77 \mu \mathrm{m}$ (рис. 7). Исследования даже

Таблица 2. Параметры ИК-активных $T_{1 u}$-фононов при $300 \mathrm{~K}$ по модели затухающих осцилляторов

\begin{tabular}{c|c|c}
\hline $\begin{array}{c}\text { Частота } \omega_{j}(\mathrm{TO}), \\
\mathrm{cm}^{-1}\end{array}$ & $\begin{array}{c}\text { Затухание } \gamma_{j}, \\
\mathrm{~cm}^{-1}\end{array}$ & $\begin{array}{c}\text { Сила осциллятора } \\
f_{j}\end{array}$ \\
\hline 132.0 & 23.9 & 1.35 \\
162.8 & 25.3 & 2.47 \\
303.0 & 42.1 & 10.2 \\
369.9 & 32.4 & 3.16 \\
510.2 & 66.6 & 1.63 \\
557.5 & 29.9 & 0.37
\end{tabular}

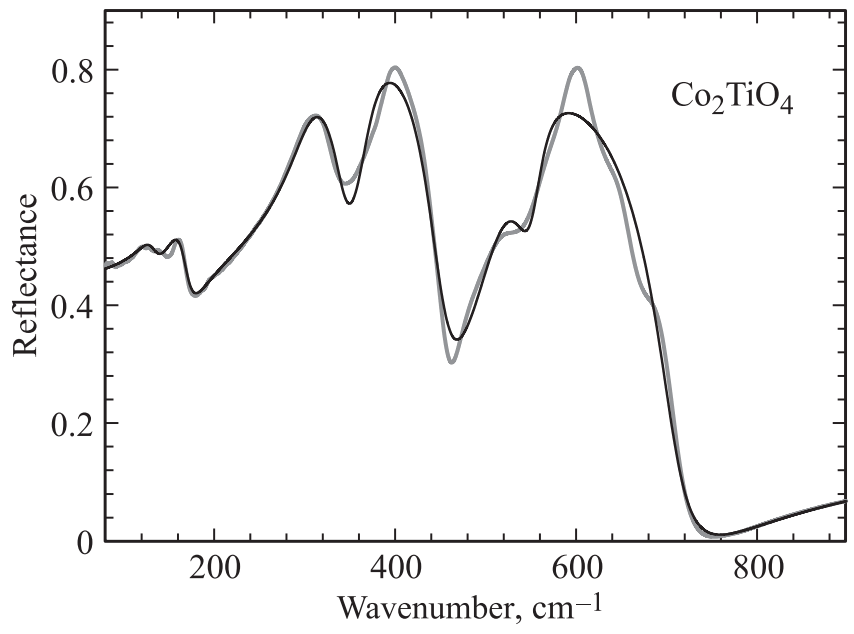

Рис. 6. Спектры ИК-отражения при температуре $300 \mathrm{~K}$. Экспериментальные данные показаны серой линией, темная линия - результаты расчета по модели затухающих осцилляторов согласно (2).

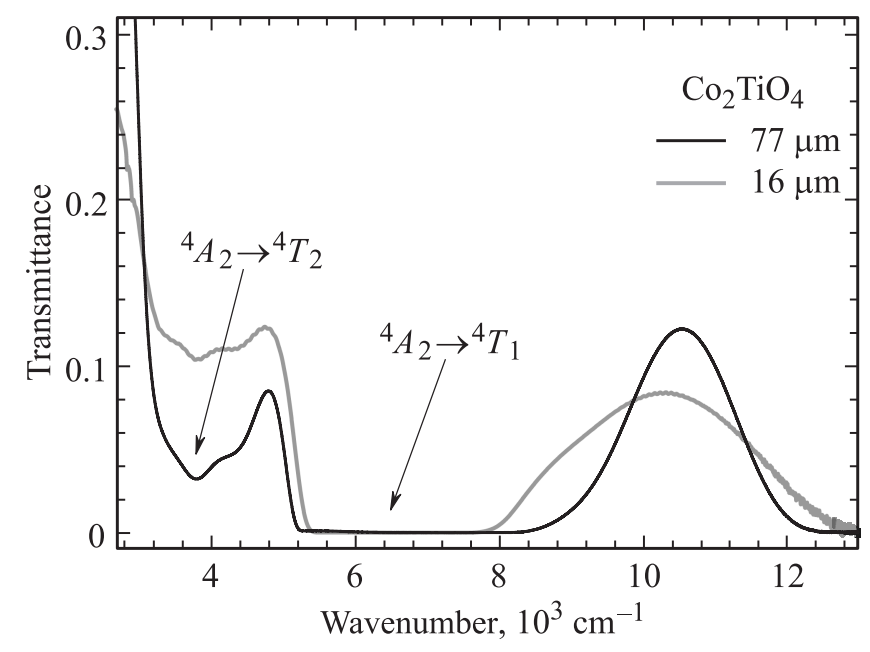

Рис. 7. Спектры пропускания в области электронных переходов при комнатной температуре на кристаллах толщиной 16 и $77 \mu \mathrm{m}$.

на столь тонких образцах не позволили полностью прописать полосы поглощения в районе 3800 и $6300 \mathrm{~cm}^{-1}$. Эти значения очень приблизительны, но тем не менее соответствующие полосы следует отнести к переходам ${ }^{4} A_{2} \rightarrow{ }^{4} T_{2}$ и ${ }^{4} A_{2} \rightarrow{ }^{4} T_{1}$ в тетраэдрических позициях. В случае второго перехода, вполне возможно, имеет место перекрытие с переходом ${ }^{4} T_{1 g} \rightarrow{ }^{4} T_{2 g}$ в октаэдрических позициях, однако их разделение не представляется возможным. Электронные $d-d$-переходы для различных соединений с $3 d^{7}$-ионами подробно изучались методами оптической спектроскопии, и типичные интервалы энергий переходов для ионов $\mathrm{Co}^{2+}$ в тетраэдрических и октаэдрических позициях показаны на рис. $2[12,18]$. В качестве примера можно указать переходы для $\mathrm{Co}^{2+}{ }_{\text {в }}$ кислородном октаэдре в вольфрамате $\mathrm{CoWO}_{4}$, в котором наблюдались переходы на уровни ${ }^{4} T_{2 g}\left(\sim 6000 \mathrm{~cm}^{-1}\right)$, 
${ }^{4} A_{2 g} \quad\left(\sim 14000 \mathrm{~cm}^{-1}\right) \quad$ и ${ }^{4} T_{g}\left({ }^{4} P\right) \quad\left(\sim 17000 \mathrm{~cm}^{-1}\right) \quad[19]$. Мы провели исследование поглощения в кристаллах $\mathrm{Co}_{3}\left(\mathrm{BO}_{3}\right)_{2}$, в которых ионы $\mathrm{Co}^{2+}$ находятся в октаэдрических позициях. Были зарегистрированы полосы поглощения в видимой области 13900 и $19200 \mathrm{~cm}^{-1}$, которые, согласно рассматриваемой схеме (рис. 2), можно отнести к переходам ${ }^{4} T_{1 g} \rightarrow{ }^{4} A_{2 g}$ и ${ }^{4} T_{1 g} \rightarrow{ }^{4} T_{2 g}\left({ }^{4} P\right)$ соответственно.

Высокая интенсивность $d-d$-переходов в $\mathrm{Co}_{2} \mathrm{TiO}_{4}$ затрудняет точное определение края интенсивного фундаментального поглощения. Как отмечалось выше [11], соединение $\mathrm{Co}_{2} \mathrm{TiO}_{4}$ является коммерческим зеленым пигментом, т.е. можно предполагать, что истинный край интенсивного поглощения лежит выше зеленой области спектра, т.е. выше 2.3-2.4 eV. В работе [10] для объемного кристалла приводится значение $2.03 \mathrm{eV}$. Наши исследования отражения также указывают на рост поглощения при энергиях выше $\sim 2.0 \mathrm{eV}$. Этот вопрос несомненно требует дальнейших исследований, например, с использованием метода спектроскопической эллипсометрии.

4.3. Диэлектрические измерения. Температурные зависимости диэлектрической проницаемости $\varepsilon(T)$, измеренные в диапазоне частот $10 \mathrm{kHz}-1 \mathrm{MHz}$ в интервале $4.2-350 \mathrm{~K}$, приведены на рис. 8. Особенности поведения температурной зависимости диэлектрической проницаемости в диапазоне от 4.2 до $200 \mathrm{~K}$ на частоте $500 \mathrm{kHz}$ приведены на вставке к рис. 8. Необычным в поведении диэлектрической проницаемости является широкий минимум в диапазоне $120-130 \mathrm{~K}$, а также ее рост при понижении температуры до $4 \mathrm{~K}$. Относительные изменения $\Delta \varepsilon$ при этом составили $\sim 0.6 \%$. При температурах Кюри $T_{C}=56 \mathrm{~K}$ и магнитной компенсации (вблизи $30 \mathrm{~K}$ ) особенности на зависимости $\varepsilon(T)$ выявлены не были. При температурах выше 120-130 K диэлектрическая проницаемость испытывает существенный рост вплоть до $350 \mathrm{~K}$. Дисперсия поведения $\varepsilon(T)$, т.е. ее зависимость от частоты, наблюдается лишь при температурах выше $200 \mathrm{~K}$ и вызвана существенным ростом проводимости. Такое поведение при повышении температуры типично для оксидов переходных металлов. Абсолютное значение $\varepsilon=21.9$ на частоте $500 \mathrm{kHz}$ при комнатной температуре несколько отличается от значения $\varepsilon_{0}=25.07$, полученного из модельного расчета спектров ИК-фононов (рис. 9).

Следует особо остановиться на росте $\varepsilon(T)$ в $\mathrm{Co}_{2} \mathrm{TiO}_{4}$ при понижении температуры. Такое явление хорошо известно в сегнетоэлектрических и диэлектрических кристаллах [20]. Модельным примером служит $\mathrm{SrTiO}_{3}$, в котором это явление получило название квантового параэлектричества [21]. Конечно, в материалах типа $\mathrm{SrTiO}_{3}$ как абсолютная величина диэлектрической проницаемости, так и ее температурные изменения существенно больше, но важен сам факт ее роста при понижении температуры. В магнитных диэлектриках лишь совсем недавно подобное явление было обнаружено в гексаферрите бария $\mathrm{BaFe}_{12} \mathrm{O}_{19}$ [22]. Наблюдаемое нами явление

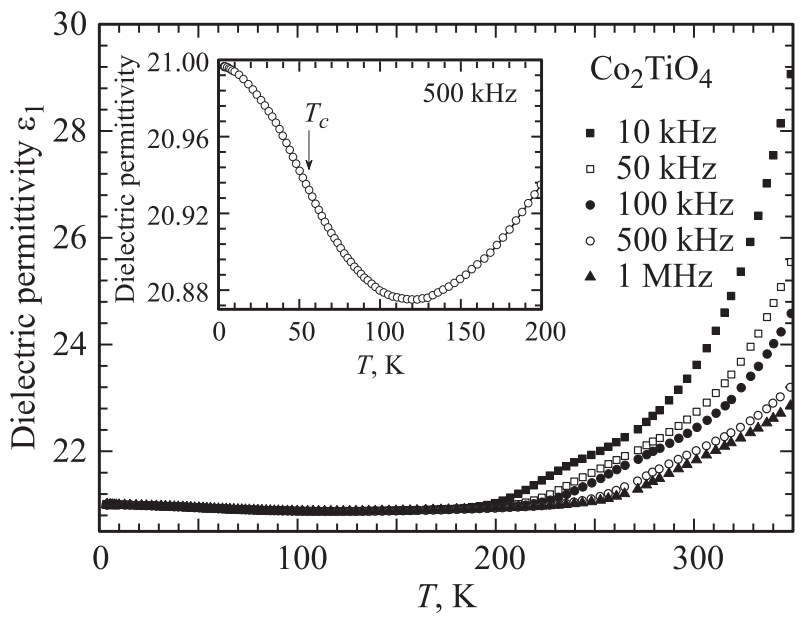

Рис. 8. Зависимости диэлектрической проницаемости $\varepsilon(T)$ от температуры для различных частот. На вставке показана область, в которой наблюдается рост $\varepsilon(T)$ при понижении температуры.

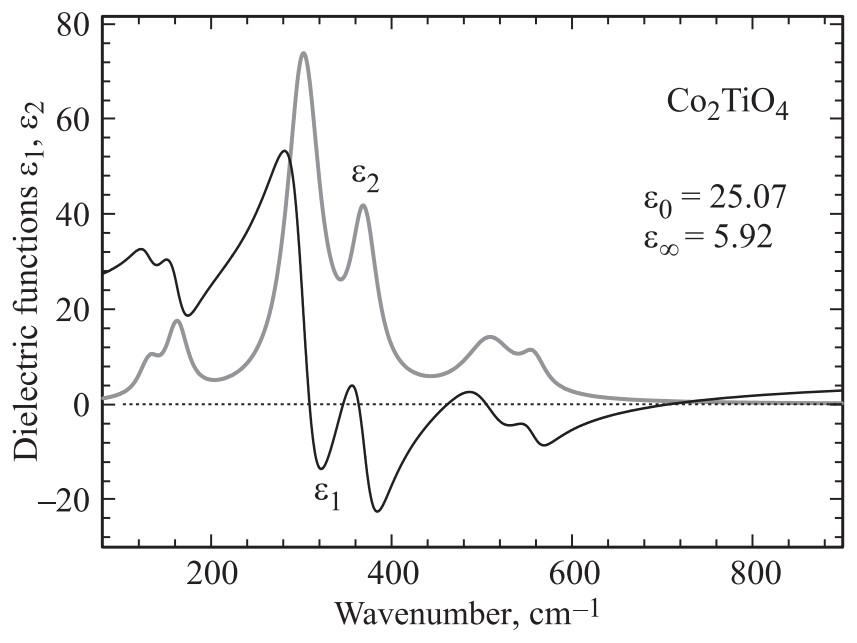

Рис. 9. Частотная зависимость диэлектрических функций $\varepsilon_{1}, \varepsilon_{2}$, рассчитанная из спектров отражения на основе (2).

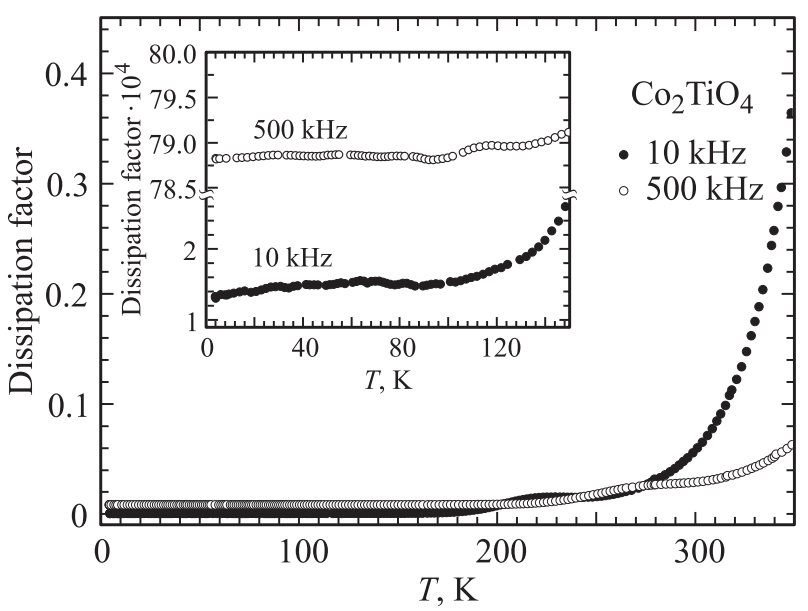

Рис. 10. Зависимости тангенса угла диэлектрических потерь от температуры для двух частот. На вставке приведена зависимость для области низких температур. 
роста $\varepsilon(T)$ в ферримагнетике $\mathrm{Co}_{2} \mathrm{TiO}_{4}$ при понижении температуры можно объяснить в рамках модели Барретта [20] (с соответствующим учетом масштабного фактора). Оно представляет, на наш взгляд, большой интерес и заслуживает дальнейшего экспериментального и теоретического исследования как в $\mathrm{Co}_{2} \mathrm{TiO}_{4}$, так и в других магнитных диэлектриках.

На рис. 10 приведены зависимости тангенса угла диэлектрических потерь $\operatorname{tg} \delta$ на частотах 10 и $500 \mathrm{kHz}$ в диапазоне от температуры жидкого азота до $350 \mathrm{~K}$. При повышении температуры от 4.2 до $100 \mathrm{~K}$ значения $\operatorname{tg} \delta$ изменяются незначительно (см. вставку на рис. 10). Эти значения при $T=4.2 \mathrm{~K}$ равны $1.34 \cdot 10^{-4}$ и $7.88 \cdot 10^{-3}$ на частотах 10 и $500 \mathrm{kHz}$ соответственно. При нагреве от 100 до $220 \mathrm{~K}$ наблюдается незначительный рост диэлектрических потерь на частоте $10 \mathrm{kHz}$; в диапазоне от 220 до $250 \mathrm{~K}$ значения $\operatorname{tg} \delta$ фактически не изменяются. Далее при повышении температуры вплоть до максимальной температуры измерений наблюдается значительный рост проводимости. На частоте $500 \mathrm{kHz}$ тангенс угла диэлектрических потерь при повышении температуры от 100 до $270 \mathrm{~K}$ растет, затем в диапазоне от 270 до $300 \mathrm{~K}$ не изменяется, далее (при нагреве вплоть до $350 \mathrm{~K}$ ) происходит рост проводимости. Абсолютные значения при температуре $350 \mathrm{~K}$ достигают значения $\operatorname{tg} \delta=0.388$ на частоте $10 \mathrm{kHz}$ и $\operatorname{tg} \delta=0.062$ на частоте $500 \mathrm{kHz}$.

\section{5. Заключение}

Методами ИК спектроскопии и комбинационного рассеяния изучена динамика решетки монокристаллов $\mathrm{Co}_{2} \mathrm{TiO}_{4}$, идентифицированы Раман-активные фононы с частотами $187.4,314.2,481.4,535.2,704.2 \mathrm{~cm}^{-1}$ в согласии с данными по шпинели $\mathrm{Fe}_{3} \mathrm{O}_{4}$. На основе спектров отражения вычислены параметры ИК-активных фононов симметрии $T_{1 u}$ и диэлектрические функции в области частот $90-900 \mathrm{~cm}^{-1}$. В области высоких частот обнаружены электронные переходы ${ }^{4} A_{2} \rightarrow{ }^{4} T_{2}$ и ${ }^{4} A_{2} \rightarrow{ }^{4} T_{1}$ ионов $\mathrm{Co}^{2+}$ в тетраэдрических позициях $8 a$. Измерены температурные зависимости диэлектрической проницаемости, согласующиеся с данными спектроскопии ИК-отражения. Обнаружен рост диэлектрической проницаемости при понижении температуры ниже $200 \mathrm{~K}$.

Авторы благодарят Л.Д. Исхакову (ЦВО РАН) за проведение рентгеновского энергодисперсионного анализа и рентгенодифракционных исследований монокристалла.

\section{Список литературы}

[1] K.E. Sickafus, J.M. Wills, N.W. Grimes. J. Am. Ceram. Soc. 82, 3279 (1999).

[2] S. Krupička, P. Novak. Handbook of ferromagnetic materials / Ed. E.P. Wohlfarth. North-Holland, Amsterdam (1982). V. 3. P. 189.
[3] S. Chikazumi. Physics of ferromagnetism. 2nd ed. Oxford University Press (2009) 682 p.

[4] Landolt-Bornstein. Magnetic oxides and related compounds. Springer, Berlin (1970) V. 3/4b.

[5] Handbook of physical quantities / Eds I.S. Grigoriev, E.Z. Meilikhov. CRC press, Boca Raton, FL (1997). Ch. 29.

[6] N. Sakamoto. J. Phys. Soc. Jpn. 17, 99 (1962).

[7] J. Hubsch, G. Gavoille. Phys. Rev. B 26, 3815 (1982).

[8] S. Nayak, S. Thota, D.C. Joshi, M. Krautz, A. Waske, A. Behler, J. Eckert, T. Sarkar, M.S. Andersson, R. Mathieu V. Narang, M.S. Seehra. Phys. Rev. B 92, 214434 (2015).

[9] S. Nayak, D.C. Joshi, M. Krautz, A. Waske, J. Eckert, S. Thota. J. Appl. Phys. 119, 043901 (2016).

[10] S. Khanahmadzadeh, M. Enhessari, Z. Solati, A. Mohebalizadeh, A. Alipouramjad. Mater. Sci. Semicond. Proc. 31, 599 (2015).

[11] T. Masui, N. Takeuchi, H. Nakado, N. Imanaka. Dyes Pigments 113, 336 (2015).

[12] R.G. Burns. Mineralogical applications of crystal field theory. 2nd ed. Cambridge University Press (1993). 576 p.

[13] O.N. Shebanova, P. Lazor. J. Solid State Chem. 174, 424 (2003).

[14] R. Bujakiewicz-Korońska, L. Hetmańczyk, B. Garbarz-Glos, A. Budziak, A. Kalvane, K. Bormanis, K. Drużbicki. Cent. Eur. J. Phys. 10, 1137 (2012).

[15] D.L.A. De Faria, S. Venâncio Silva, M.T. De Oliveira. J. Raman Spectrosc. 28, 873 (1997).

[16] M. Balkanski, R.F. Wallis, E. Haro. Phys. Rev. B 28, 1928 (1983).

[17] A.B. Kuzmenko. Rev. Sci. Instrum. 76, 083108 (2005).

[18] A.B.P. Lever. Inorganic electronic spectroscopy. 2nd ed. Elsevier (1968). 420 p.

[19] J. Ferguson, D.L. Wood, K. Knox. J. Chem. Phys. 39, 881 (1963).

[20] J.H. Barrett. Phys. Rev. 86, 118 (1952).

[21] K.A. Müller, H. Burkard. Phys. Rev. B 19, 3593 (1979).

[22] S.-P. Shen, J.-C. Wu, J.-D. Song, X.-F. Sun, Y.-F. Yang, Y.-S. Chai, D.-S. Shang, S.-G. Wang, J.F. Scott, Y. Sun. Nature Commun. 7, 10569 (2016). 